\title{
Synthesis, characterisation and biological screening of $s$ - triazine based chalcones and its derivatization into phenyl pyrazolines, isoxazoles
}

\author{
Anjani Solankee*, Riki Tailor \\ Department of Chemistry, B. K. M. Science College, Valsad - 396001 \\ (Affiliated to The Veer Narmad South Gujarat University, Surat) India \\ *E-mail address: dranjani_solankee@yahoo.com
}

\begin{abstract}
Heterocyclic derivatives such as phenyl pyrazolines and isoxaxoles were prepared from $s$ triazine based chalcones. Chalcones $\left(\mathrm{A}_{1}-\mathrm{A}_{5}\right)$ are synthesised by the reaction of compound $(\mathrm{V})$ with various aromatic aldehydes. Moreover, further reaction of chalcones with phenyl hydrazine hydrochloride and hydroxylamine hydrochloride in the presence of alkali gives phenyl pyrazolines $\left(\mathrm{A}_{6}\right.$ - $\left.A_{10}\right)$ and isoxazoles $\left(A_{11}-A_{15}\right)$ derivatives respectively. The structures of the newly synthesised compounds were confiremed by spectroscopic (IR, ${ }^{1} \mathrm{H}$ NMR, ${ }^{13} \mathrm{C}$ NMR) and elemental analysis. All the newly synthesised compounds have been screened for their antimicrobial activity against selected Gram - positive (S. aureus and S. pyogenus), Gram - negative (E. coli and P. aeruginosa) bacterial and fungal strains (C. albicans, A. niger and A. clavatus).
\end{abstract}

Keywords: Chalcones; phenyl pyarazolines; isoxazoles; spectral data; elemental analysis; antimicrobial activity

\section{INTRODUCTION}

The main fears for human beings are a variety of diseases. Scientists and doctors are still struggling to find solutions with various forms of medications. Today's new medicines are results of inexorable effort made by human civilization time to time. The most common compounds of chalconoid group are the chalcones, which provide new class of medicines due to the pharmacologically active moiety and various biological activities. The chalcones are 1 , 3 - diarylprop - 2 - en - 1 - one, form a broad class of compounds containing two aromatic rings bound with vinyl ketone fragment. Chalcones are useful intermediates for obtaining the variety of heterocycles [1-5]. Various chalcone derivatives are remarkable materials for their second harmonic generation [6]. They are naturally occurring plant metabolites possess a broad spectrum of biological activities such as cancer cell lines [7-8], antimitotic [9], antiinflammatory [10], hepatoprotective [11], molluscicidal properties [12], heme oxygenase-1 [13], antimicrobial [14] etc... . So, this broad spectrum of applications encouraged us to search for another addition to the existed molecule. 
Pyrazoline derivatives with a phenyl group at 5 - position show good film- forming properties, excellent features of blue photoluminescence and electroluminescence [15]. Pyrazoline derivatives have a long history of application in agrochemicals and pharmaceutical industry as herbicides and active pharmaceuticals. Now days, a major portion of research in heterocyclic chemistry has been devoted to 2- pyrazolines containing diverse aryl groups as substituents. Pyrazoline derivatives are well known for their different biological activities such as antifeedant [16], anti- inflammatory [17], antiviral [18], antidepressant [19], antibacterial [20], antifungal [21] etc... . Many class of chemotherapeutic agents containing pyrazoline nucleus are in clinical use such as orisul (antibacterial), antipyrine (antipyretic), butazolidine (anti-inflammatory). So based on the above biological activities exhibited by the pyrazoline compounds, we reported here, the synthesis and biological screening of some novel phenyl pyrazoline derivatives.

Among heterocycles, the isoxazole unit constitutes an easily accessible nucleus that is present in a number of natural and pharmacological compounds [22], display a wide range of organic reactivities and used as an effective means of preparing new molecular scaffolds [23]. Isoxazoles have been repeatedly shown as useful synthons in organic synthesis [24]. Isoxazoles shows a broad spectrum of biological properties like fungicidal [25], antimicrobial [26], antitubercular [27], antiviral [28] etc... . So in this regard, we have synthesised some novel isoxazole derivatives and screened this compounds to antimicrobial activitiy.

\section{EXPERIMENTAL}

\subsection{Material}

All the chemicals and solvents which used for reaction were purified after getting from commercial suppliers. Melting points were taken in open capillaries using paraffin bath and were uncorrected. IR spectra were recorded on Shimadzu IR Affinity - 1 FTIR spectrometer $\left(\mathrm{V}_{\max }-1\right),{ }^{1} \mathrm{H}$ NMR were recorded on Bruker Avance - DPX $400 \mathrm{MHz}$ NMR spectrometer using $\mathrm{CDCl}_{3}$ as a solvent and TMS as internal reference and ${ }^{13} \mathrm{C}$ NMR spectra were recorded on the same instrument at $100 \mathrm{MHz}$ operating frequency using DMSO as a solvent and TMS as internal reference. The chemical shifts are expressed in parts per million (ppm) downfield from the internal standard and signals are quoted as s (singlet), $\mathrm{d}$ (doublet) and $\mathrm{m}$ (multiplate). The coupling constants $(J)$ are given in Hertz $(\mathrm{Hz})$. All the compounds were analyzed for carbon, hydrogen and nitrogen by the Perkin-Elmer $240 \mathrm{C}$ $\mathrm{H} \mathrm{N}$ elemental analyzer and the results were within $\pm 0.4 \%$ of theoretical values. The purity of synthesised compounds were checked by thin layer chromatography conducted on Silica Gel 60 F-254 (Merck) plates of $0.25 \mathrm{~mm}$ thickness and the spots were located using toluene : methanol $(12: 6 \mathrm{v} / \mathrm{v})$ eluents and visualized with UV $(254 \mathrm{~nm})$ light or keeping the plates in iodine chamber.

\subsection{Method}

\subsection{A. General procedure for the compounds (III), (IV) and (V)}

Compounds (III), (IV) and (V) were prepared by the reported method [29]. 


\subsection{B. Preparation of 2 - (3' - trifluromethylphenylamino) - 4 - (tetrahydro - 1' , 4' - oxazine $)-6-\left[4^{\prime}-\left\{3^{\prime \prime}-\left(4^{\prime \prime \prime}-\right.\right.\right.$ methoxyphenyl $)-2^{\prime \prime}-$ propenon - 1" - yl $\}$ phenylamino] - $s$ - triazine $\left(A_{1}\right)$}

In a round- bottomed flask, substituted acetophenone (V) $(0.01 \mathrm{~mol}, 4.5 \mathrm{~g}$ in $20 \mathrm{ml}$ DMF) was dissolved in a dimethyl formamide and 4 - methoxybenzaldehyde $(0.01 \mathrm{~mol}, 1.36 \mathrm{~g}$ in $10 \mathrm{ml}$ DMF) was added in it. To make this mixture alkaline $40 \% \mathrm{KOH}(5 \mathrm{ml})$ was added as catalyst, then the reaction mixture was stirred for 24 hours at room temprature. The progress of reaction was monitored by TLC. After completion of the reaction, the reaction mixture was poured into crushed ice and neutralise with $\mathrm{HCl}$. Finally, the product was filtered, dried and purified by recrystallization from ethanol. In the same way, the remaining compounds $\left(\mathbf{A}_{\mathbf{2}}-\right.$ A5) were prepared by this method. All the synthesised compounds ( $\left.\mathbf{A}_{\mathbf{1}}-\mathbf{A}_{\mathbf{5}}\right)$ were characterised by IR, ${ }^{1} \mathrm{H}$ NMR, ${ }^{13} \mathrm{C}$ NMR spectroscopy, the characteristic data of the entire synthesised compounds are given in spectral analysis data (3) and physical data are given in Table - 1.

2.2.C. 2 - (3' - trifluromethylphenylamino) - 4 - (tetrahydro - 1', 4' - oxazine) - 6 - [4' $\left\{1^{\prime \prime}-\right.$ phenyl 5"'- (4"' - methoxyphenyl) $2^{\prime \prime}$ - pyrazolin $\left.3^{\prime \prime}-\mathrm{yl}\right\}$ phenylamino] - s triazine $\left(\mathbf{A}_{6}\right)$

chalcone $\left(\mathbf{A}_{\mathbf{1}}\right)$ (0.01 mol, 5.76g in $30 \mathrm{ml}$ alcohol) and phenyl hydrazine hydrochloride $(0.01 \mathrm{~mol}, 1.44 \mathrm{~g}$ in $10 \mathrm{ml}$ alcohol) was dissolved in alcohol. To make this mixture alkaline $40 \% \mathrm{KOH}(5 \mathrm{ml})$ was added to the reaction mixture and refluxed for 8 hours. The progress of the reaction was monitored by using TLC. After completion of the reaction, the reaction mixture was poured into crushed and neutralise with dilute $\mathrm{HCl}$, Finally, the product was filtered, washed with water, dried and recrystallization from ethanol to get product $\left(\mathbf{A}_{\mathbf{6}}\right)$ in good yield with high purity. In the same way other remaining compounds $\left(\mathbf{A}_{7}-\mathbf{A}_{10}\right)$ were prepared by this method. All the synthesised compounds $\left(\mathbf{A}_{\mathbf{6}}-\mathbf{A}_{\mathbf{1 0}}\right)$ were characterised by IR, ${ }^{1} \mathrm{H}$ NMR, ${ }^{13} \mathrm{C}$ NMR spectroscopy, the characteristic data of the entire synthesised compounds are given in spectral analysis data (3) and physical data are given in Table - $\mathbf{1}$.

2.2.D. Preparation of 2 - (3' - trifluromethyphenylamino) - 4 - (tetrahydro - 1', 4' oxazine) - 6 - [4' - \{5"'- (4"' - methoxyphenyl) $2^{\prime \prime}$ - isoxazol - 3"'-yl\} phenylamino] - $s$ - triazine $\left(A_{11}\right)$

Compound ( $\left.\mathbf{A}_{1}\right)(0.01 \mathrm{~mol}, 5.76 \mathrm{~g}$ in $30 \mathrm{ml}$ alcohol$)$ and hydroxylamine hydrochloride $(0.01 \mathrm{~mol}, 0.695 \mathrm{~g}$ in $10 \mathrm{ml}$ alcohol $)$ was dissolved in methanol. To make this mixture alkaline $40 \% \mathrm{KOH}(5 \mathrm{ml})$ was added to the reaction mixture and refluxed for 8 hours. The progress of the reaction was monitored by using TLC. After completion of the reaction, the reaction mixture was poured into crushed ice and neutralised with dilute $\mathrm{HCl}$. Finally, the product was filtered, washed with water, dried and recrystallization from ethanol to get product $\left(\mathbf{A}_{11}\right)$ in good yield with high purity. In the same way other remaining compounds $\left(\mathbf{A}_{12}-\mathbf{A}_{15}\right)$ were prepared by this method. All the synthesised compounds $\left(\mathbf{A}_{12}-\mathbf{A}_{15}\right)$ were characterised by IR, ${ }^{1} \mathrm{H}$ NMR, ${ }^{13} \mathrm{C}$ NMR spectroscopy, the characteristic data of the entire synthesised compounds are given in spectral analysis data (3) and physical data are given in Table - $\mathbf{1}$. 


\subsection{Reaction Scheme}<smiles>Clc1nc(Cl)nc(Cl)n1</smiles>

I<smiles>Nc1cccc(C(F)(F)F)c1</smiles>

II

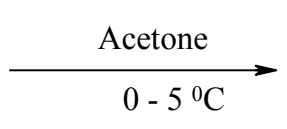<smiles>C1COCCN1</smiles>

Acetone Room Temp.<smiles>CC(=O)c1ccc(Nc2nc(Nc3cccc(C(F)(F)F)c3)nc(N3CCOCC3)n2)cc1</smiles>

V

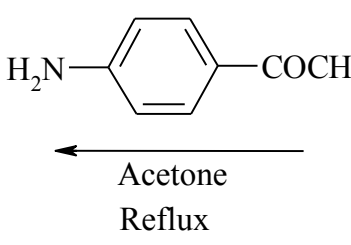
Reflux

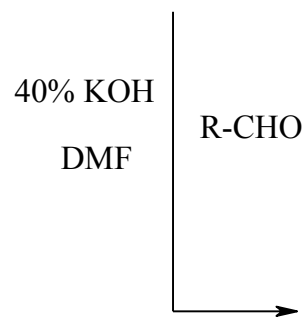<smiles>[R]C=CC(=O)c1ccc(Nc2nc(Nc3cccc(C(F)(F)F)c3)nc(N3CCOCC3)n2)cc1</smiles><smiles>[R]c1cc(-c2ccc(Nc3nc(Nc4cccc(C(F)(F)F)c4)nc(N4CCOCC4)n3)cc2)no1</smiles>

$A_{11}-A_{15}$<smiles>[R]C1CC(c2ccc(Nc3nc(Nc4cccc(C(F)(F)F)c4)nc(N4CCOCC4)n3)cc2)=NN1c1ccccc1</smiles> 
Table 1. The physical data of synthesised compounds $A_{1}-A_{15}$.

\begin{tabular}{|c|c|c|c|c|c|c|c|}
\hline \multirow[t]{2}{*}{ Compd } & \multirow[t]{2}{*}{$\mathbf{R}$} & \multirow[t]{2}{*}{ Molecular Formula } & \multirow[t]{2}{*}{$\begin{array}{l}\text { Yield } \\
(\%)\end{array}$} & \multirow[t]{2}{*}{$\underset{{ }^{\mathbf{M}} \mathrm{C}}{\mathrm{M}}$} & \multicolumn{3}{|c|}{$\begin{array}{c}\text { Elemental analysis } \\
\text { Calculated } \\
\text { (Found) } \% \\
\end{array}$} \\
\hline & & & & & $\mathbf{C}$ & $\mathbf{H}$ & $\mathbf{N}$ \\
\hline $\mathrm{A}_{1}$ & 4 - Methoxy phenyl & $\mathrm{C}_{30} \mathrm{H}_{27} \mathrm{~N}_{6} \mathrm{~F}_{3} \mathrm{O}_{3}$ & 74 & 180 & $\begin{array}{c}62.50 \\
(62.55)\end{array}$ & $\begin{array}{c}4.72 \\
(4.73)\end{array}$ & $\begin{array}{c}14.58 \\
(14.61)\end{array}$ \\
\hline $\mathrm{A}_{2}$ & 4 - Chloro phenyl & $\mathrm{C}_{29} \mathrm{H}_{24} \mathrm{~N}_{6} \mathrm{~F}_{3} \mathrm{O}_{2} \mathrm{Cl}$ & 69 & 125 & $\begin{array}{c}59.95 \\
(59.96)\end{array}$ & $\begin{array}{c}4.16 \\
(4.20)\end{array}$ & $\begin{array}{c}14.47 \\
(14.45)\end{array}$ \\
\hline $\mathrm{A}_{3}$ & 3 - Phenoxyphenyl & $\mathrm{C}_{35} \mathrm{H}_{29} \mathrm{~N}_{6} \mathrm{~F}_{3} \mathrm{O}_{3}$ & 71 & 148 & $\begin{array}{c}65.83 \\
(65.89)\end{array}$ & $\begin{array}{c}4.57 \\
(4.60) \\
\end{array}$ & $\begin{array}{c}13.16 \\
(13.12)\end{array}$ \\
\hline $\mathrm{A}_{4}$ & 2 - Nitrophenyl & $\mathrm{C}_{29} \mathrm{H}_{24} \mathrm{~N}_{7} \mathrm{~F}_{4} \mathrm{O}_{3}$ & 70 & 105 & $\begin{array}{c}58.88 \\
(58.84) \\
\end{array}$ & $\begin{array}{c}4.09 \\
(4.05) \\
\end{array}$ & $\begin{array}{c}16.58 \\
(16.55) \\
\end{array}$ \\
\hline $\mathrm{A}_{5}$ & Phenyl & $\mathrm{C}_{29} \mathrm{H}_{25} \mathrm{~N}_{6} \mathrm{~F}_{3} \mathrm{O}_{2}$ & 65 & 108 & $\begin{array}{c}63.73 \\
(63.70)\end{array}$ & $\begin{array}{c}4.61 \\
(4.65)\end{array}$ & $\begin{array}{c}15.38 \\
(15.43)\end{array}$ \\
\hline $\mathrm{A}_{6}$ & 4 - Methoxy phenyl & $\mathrm{C}_{36} \mathrm{H}_{33} \mathrm{~N}_{8} \mathrm{~F}_{3} \mathrm{O}_{2}$ & 72 & 165 & $\begin{array}{c}64.86 \\
(64.83)\end{array}$ & $\begin{array}{c}4.98 \\
(4.95)\end{array}$ & $\begin{array}{c}16.81 \\
(16.79)\end{array}$ \\
\hline $\mathrm{A}_{7}$ & 4 - Chloro phenyl & $\mathrm{C}_{35} \mathrm{H}_{30} \mathrm{~N}_{8} \mathrm{~F}_{3} \mathrm{OCl}$ & 69 & 98 & $\begin{array}{c}62.64 \\
(62.83) \\
\end{array}$ & $\begin{array}{c}4.50 \\
(4.53) \\
\end{array}$ & $\begin{array}{c}16.70 \\
(16.72) \\
\end{array}$ \\
\hline $\mathrm{A}_{8}$ & 3 - Phenoxyphenyl & $\mathrm{C}_{41} \mathrm{H}_{35} \mathrm{~N}_{8} \mathrm{~F}_{3} \mathrm{O}_{2}$ & 68 & 141 & $\begin{array}{c}67.57 \\
(67.55)\end{array}$ & $\begin{array}{c}4.84 \\
(4.87)\end{array}$ & $\begin{array}{c}15.38 \\
(15.43)\end{array}$ \\
\hline $\mathrm{A}_{9}$ & 2 - Nitrophenyl & $\mathrm{C}_{35} \mathrm{H}_{30} \mathrm{~N}_{9} \mathrm{~F}_{3} \mathrm{O}_{3}$ & 67 & 130 & $\begin{array}{c}61.67 \\
(61.70)\end{array}$ & $\begin{array}{c}4.43 \\
(4.46)\end{array}$ & $\begin{array}{c}18.49 \\
(14.47)\end{array}$ \\
\hline $\mathrm{A}_{10}$ & Phenyl & $\mathrm{C}_{35} \mathrm{H}_{31} \mathrm{~N}_{8} \mathrm{~F}_{3} \mathrm{O}$ & 70 & 94 & $\begin{array}{c}66.03 \\
(66.01)\end{array}$ & $\begin{array}{c}4.90 \\
(4.87)\end{array}$ & $\begin{array}{c}17.60 \\
(16.58)\end{array}$ \\
\hline $\mathrm{A}_{11}$ & 4 - Methoxy phenyl & $\mathrm{C}_{30} \mathrm{H}_{26} \mathrm{~N}_{7} \mathrm{~F}_{3} \mathrm{O}_{3}$ & 74 & 109 & $\begin{array}{l}61.12 \\
(61.1)\end{array}$ & $\begin{array}{c}4.44 \\
(4.47)\end{array}$ & $\begin{array}{c}16.63 \\
(14.62)\end{array}$ \\
\hline $\mathrm{A}_{12}$ & 4 - Chloro phenyl & $\mathrm{C}_{29} \mathrm{H}_{23} \mathrm{~N}_{7} \mathrm{~F}_{3} \mathrm{O}_{2} \mathrm{Cl}$ & 65 & 155 & $\begin{array}{c}58.64 \\
(58.66) \\
\end{array}$ & $\begin{array}{c}3.90 \\
(3.87)\end{array}$ & $\begin{array}{c}16.51 \\
(16.53) \\
\end{array}$ \\
\hline $\mathrm{A}_{13}$ & 3 - Phenoxyphenyl & $\mathrm{C}_{35} \mathrm{H}_{28} \mathrm{~N}_{7} \mathrm{~F}_{3} \mathrm{O}_{3}$ & 71 & 123 & $\begin{array}{c}64.51 \\
(64.47)\end{array}$ & $\begin{array}{c}4.33 \\
(4.31)\end{array}$ & $\begin{array}{c}15.05 \\
(15.04)\end{array}$ \\
\hline $\mathrm{A}_{14}$ & 2 - Nitrophenyl & $\mathrm{C}_{29} \mathrm{H}_{23} \mathrm{~N}_{8} \mathrm{~F}_{3} \mathrm{O}_{4}$ & 73 & 117 & $\begin{array}{c}57.62 \\
(57.59)\end{array}$ & $\begin{array}{c}3.83 \\
(3.86)\end{array}$ & $\begin{array}{c}18.54 \\
(18.51)\end{array}$ \\
\hline $\mathrm{A}_{15}$ & Phenyl & $\mathrm{C}_{29} \mathrm{H}_{24} \mathrm{~N}_{7} \mathrm{~F}_{3} \mathrm{O}_{2}$ & 66 & 110 & $\begin{array}{c}62.25 \\
(62.21)\end{array}$ & $\begin{array}{c}4.32 \\
(4.35)\end{array}$ & $\begin{array}{c}17.52 \\
(17.55)\end{array}$ \\
\hline
\end{tabular}

\section{SPECTRAL ANALYSIS DATA}

Compound $\mathbf{A}_{1}: 2$ - (3' - trifluromethylphenylamino) - 4 - (tetrahydro - 1' , 4' - oxazine) - 6 - [4' - \{3" - (4'"- methoxyphenyl $)$ - 2" - propenon - 1" - yl $\}$ phenylamino] - s - triazine. : IR $(\mathrm{KBr}) \mathrm{cm}^{-1}: 3307$ (N-H str.), 3018 ( $\left.=\mathrm{CH}\right), 1624(-\mathrm{C}=\mathrm{O}), 1578$ (C=C str.), 1248 ( C -O- C str. ), 1025 ( C-F ), 807 ( C-N str. s - triazine ). ${ }^{1} \mathrm{H}$ NMR ( $\delta$ ppm, $\left.\mathrm{CDCl}_{3}\right): 8.25(\mathrm{~s}, 1 \mathrm{H},-\mathrm{NH}$ ), $3.71\left(\mathrm{t}, 8 \mathrm{H},-\mathrm{CH}_{2}\right.$, oxazine ring ), $3.89\left(\mathrm{~s}, 3 \mathrm{H}, \mathrm{p}-\mathrm{OCH}_{3}\right), 6.89(\mathrm{~d}, \mathrm{~J}=9.36 \mathrm{~Hz}, \mathrm{H},-\mathrm{CO}-$ $\mathrm{CH}=), 8.21(\mathrm{~d}, \mathrm{~J}=8.6 \mathrm{~Hz}, 1 \mathrm{H}, \mathrm{Ar}-\mathrm{CH}=), 6.98-8.1(\mathrm{~m}, 12 \mathrm{H}, \operatorname{Ar}-\mathrm{H}) .{ }^{13} \mathrm{C} \mathrm{NMR}(\delta \mathrm{ppm}$, DMSO ) : 48.7, 55.5, 66.3, 111.8, 115.1, 116.1, 121.1, 121.3, 124.1, 127.9, 128.7, 129.0, 129.8, 131.8, 132.0, 133.5, 133.7, 142.7, 144.7, 145.9, 159.8, 165.7, 168.9, 176.0, 189.7.

Compound $\mathbf{A}_{2}: 2$ - (3' - trifluromethylphenylamino) - 4 - (tetrahydro - 1' , 4' - oxazine) - 6 - [4' - \{3" - (4"'- chlorophenyl $)$ - 2" - propenon - 1" - yl $\}$ phenylamino $]$ - s - triazine. : IR $(\mathrm{KBr}) \mathrm{cm}^{-1}: 3323$ (N-H str.), 3001 ( $=\mathrm{CH}$ ), 1652 ( $\left.-\mathrm{C}=\mathrm{O}\right), 1569$ (C=C str.), 1232 ( C -O- C str. ), 1019 ( C- F ), 798 ( C-N str. s - triazine ), 639 ( C- Cl ). ${ }^{1} \mathrm{H}$ NMR ( $\delta$ ppm, $\mathrm{CDCl}_{3}$ ) : $8.35(\mathrm{~s}, 1 \mathrm{H},-\mathrm{NH}), 3.77$ ( t, $8 \mathrm{H},-\mathrm{CH}_{2}$, oxazine ring ), $6.13(\mathrm{~d}, \mathrm{~J}=9.8 \mathrm{~Hz}, 1 \mathrm{H},-\mathrm{CO}-\mathrm{CH}=)$, 
$7.91(\mathrm{~d}, \mathrm{~J}=9.4 \mathrm{~Hz}, 1 \mathrm{H}, \mathrm{Ar} \mathrm{CH}=), 7.0-8.2(\mathrm{~m}, 12 \mathrm{H}, \mathrm{Ar}-\mathrm{H}) .{ }^{13} \mathrm{C}$ NMR $(\delta \mathrm{ppm}, \mathrm{DMSO})$ : $48.6,63.1,109.3,110.9,112.8,113.1,120.8,121.6,125.2,125.9,127.1,129.1,130.5$, $131.8,132.7,135.0,138.2,141.8,145.7,166.0,172.4,179.3,186.3$.

Compound $\mathbf{A}_{3}: 2$ - (3' - trifluromethylphenylamino) - 4 - (tetrahydro - 1' , 4' - oxazine) - 6 - [4' - \{3" - (3"'- phenoxyphenyl) - 2" - propenon - 1" - yl $\}$ phenylamino $]$ - s - triazine. : IR $(\mathrm{KBr}) \mathrm{cm}^{-1}: 3338$ (N-H str.), 3023 ( $\left.=\mathrm{CH}\right), 1710(-\mathrm{C}=\mathrm{O}), 1530(\mathrm{C}=\mathrm{C}$ str. $), 1225$ ( C-O-C str. ), 1098 ( C-F ), 789 ( C-N str. s - triazine ). ${ }^{1} \mathrm{H}$ NMR ( $\delta$ ppm, $\left.\mathrm{CDCl}_{3}\right): 8.1(\mathrm{~s}, 1 \mathrm{H},-\mathrm{NH})$, 3.19 ( t, 8H, $-\mathrm{CH}_{2}$, oxazine ring ), $6.3(\mathrm{~d}, \mathrm{~J}=8.9 \mathrm{~Hz}, 1 \mathrm{H},-\mathrm{CO}-\mathrm{CH}=), 8.3(\mathrm{~d}, \mathrm{~J}=9.3 \mathrm{~Hz}, 1 \mathrm{H}$, $\mathrm{Ar}-\mathrm{CH}=)$, $6.9-7.8(\mathrm{~m}, 17 \mathrm{H}, \mathrm{Ar}-\mathrm{H}),{ }^{13} \mathrm{CNMR}$ ( $\delta \mathrm{ppm}$, DMSO $): 47.1,60.4,110.6,111.8$, $113.0,115.9,118.3,120.1,121.4,123.6,125.3,129.0,130.7,132.1,133.6,134.2,135.7$, $138.3,143.2,145.6,155.3,157.1,166.1,169.7,179.3,184.7$.

Compound $\mathbf{A}_{4}: 2$ - (3' - trifluromethylphenylamino) - 4 - (tetrahydro - 1' , 4' - oxazine) - 6 - [4' - \{3" - (2'"- nitrophenyl $)$ - 2" - propenon - 1" - yl $\}$ phenylamino $]$ - s - triazine. : IR $(\mathrm{KBr}) \mathrm{cm}^{-1}: 3310(\mathrm{~N}-\mathrm{H}$ str. $), 3035(=\mathrm{CH}), 1706(-\mathrm{C}=\mathrm{O}), 1540\left(\mathrm{C}-\mathrm{NO}_{2}\right), 1505(\mathrm{C}=\mathrm{C}$ str. $)$, 1240 ( C-O-C str. ), 1016 ( C-F ), 800 ( C-N str. s - triazine ), ${ }^{1} \mathrm{H}$ NMR ( $\delta$ ppm, $\left.\mathrm{CDCl}_{3}\right): 8.2$ ( s, $1 \mathrm{H},-\mathrm{NH}), 3.83\left(\mathrm{t}, 8 \mathrm{H},-\mathrm{CH}_{2}\right.$, oxazine ring ), $6.25(\mathrm{~d}, \mathrm{~J}=9.2 \mathrm{~Hz}, 1 \mathrm{H},-\mathrm{CO}-\mathrm{CH}=), 7.12($ $\mathrm{d}, \mathrm{J}=8.8 \mathrm{~Hz}, 1 \mathrm{H}, \mathrm{Ar}-\mathrm{CH}=), 6.9-7.9(\mathrm{~m}, 12 \mathrm{H}, \mathrm{Ar}-\mathrm{H}) .{ }^{13} \mathrm{C}$ NMR ( $\delta \mathrm{ppm}$, DMSO $): 43.2$, $67.6,109.5,111.2,118.0,121.2,124.8,126.0,127.5,128.0,130.2,131.5,133.1,133.2$, $134.5,141.8,143.8,147.7,166.4,168.1,175.1,188.2$.

Compound $\mathbf{A}_{5}: 2$ - (3' - trifluromethylphenylamino) - 4 - (tetrahydro - 1' , 4' - oxazine) - 6 - [4' - \{3" - (phenyl) - 2" - propenon - 1" - yl $\}$ phenylamino $-\mathrm{s}-$ triazine. : IR $(\mathrm{KBr}) \mathrm{cm}^{-1}$ : 3333 (N-H str.), 3019 ( =CH ), 1652 ( $-\mathrm{C}=\mathrm{O}$ ), 1516 (C=C str.), 1249 ( C-O-C str. ), 1065 ( C$\mathrm{F}), 806$ ( C-N str. s - triazine $).{ }^{1} \mathrm{H}$ NMR $\left(\delta \mathrm{ppm}, \mathrm{CDCl}_{3}\right): 7.9(\mathrm{~s}, 1 \mathrm{H},-\mathrm{NH}), 3.76(\mathrm{t}, 8 \mathrm{H},-$ $\mathrm{CH}_{2}$, oxazine ring $), 6.6(\mathrm{~d}, \mathrm{~J}=9.6 \mathrm{~Hz}, 1 \mathrm{H},-\mathrm{CO}-\mathrm{CH}=), 7.3(\mathrm{~d}, \mathrm{~J}=9.9 \mathrm{~Hz}, 1 \mathrm{H}, \mathrm{Ar}-\mathrm{CH}=), 7.1$ - 8.0 ( m, 13H, Ar-H ). ${ }^{13} \mathrm{CNMR}$ ( $\delta$ ppm, DMSO ) : 46.4, 66.2, 110.2, 112.1, 115.2, 116.6, $122.1,124.2,126.1,127.8,130.0,131.2,132.9,133.5,135.9$, , 142.7, 144.3, 163.0, 167.6, 175.1, 188.0 .

Compound $\mathbf{A}_{6}: 2$ - (3' - trifluromethylphenylamino) - 4 - (tetrahydro - 1', 4' - oxazine) - 6 - [4' - \{1"- phenyl 5"- (4"' - methoxyphenyl) 2" - pyrazolin 3"- yl $\}$ phenylamino] - s triazine : IR (KBr) cm $\mathrm{cm}^{-1}$ : 3265 (-NH), 3031 (=CH str.), 2912 (C-H str., pyrazoline moiety), $1645\left(\mathrm{C}=\mathrm{N}\right.$, pyrazoline moiety), $1256(\mathrm{C}-\mathrm{O}-\mathrm{C}), 1100(\mathrm{C}-\mathrm{F}) .{ }^{1} \mathrm{H}$ NMR $\left(\delta \mathrm{ppm}, \mathrm{CDCl}_{3}\right): 2.1$

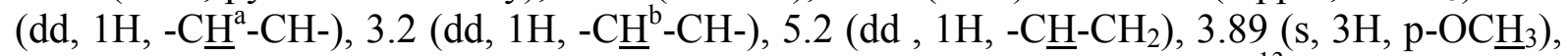
3.69 (t, $8 \mathrm{H},-\mathrm{CH}_{2}$, oxazine ring), 6.9 to $8.2(\mathrm{~m}, 18 \mathrm{H}, 17 \mathrm{Ar}-\underline{\mathrm{H}}$ and $1-\mathrm{N} \underline{\mathrm{H}}) .{ }^{13} \mathrm{C} \mathrm{NMR}(\delta \mathrm{ppm}$, DMSO) : 40.6, 46.9, 57.1, 64.3, 66.3, 110.8, 112.1, 114.0, 115.6, 120.7, 123.9, 127.1, 130.0, $131.5,133.6,134.7,140.4,142.6,144.8,150.7,157.9,162.4,164.1,174.1$.

Compound $\mathbf{A}_{7}: 2$ - (3' - trifluromethylphenylamino) - 4 - (tetrahydro - 1', 4' - oxazine) - 6 - [4' - \{1"- phenyl 5"- (4"' - chlorophenyl) 2" - pyrazolin 3"- yl $\}$ phenylamino] - s - triazine : IR (KBr) cm $\mathrm{cm}^{-1}: 3146(-\mathrm{NH}), 3054$ (=CH str.), 2936 (C-H str., pyrazoline moiety), 1640 $\left(\mathrm{C}=\mathrm{N}\right.$, pyrazoline moiety), $1235(\mathrm{C}-\mathrm{O}-\mathrm{C}), 1067(\mathrm{C}-\mathrm{F}), 663(\mathrm{C}-\mathrm{Cl}) .{ }^{1} \mathrm{H}$ NMR $\left(\delta \mathrm{ppm}, \mathrm{CDCl}_{3}\right)$ : 2.6 (dd, $\left.1 \mathrm{H},-\mathrm{CH}^{\mathrm{a}}-\mathrm{CH}-\right), 2.9$ (dd, $\left.1 \mathrm{H},-\mathrm{CH}^{\mathrm{b}}-\mathrm{CH}-\right), 5.1$ (dd, $\left.1 \mathrm{H},-\mathrm{C} \underline{\mathrm{H}}-\mathrm{CH}_{2}\right), 3.10\left(\mathrm{t}, 8 \mathrm{H},-\mathrm{CH}_{2}\right.$, oxazine ring), 7.0 to $8.2(\mathrm{~m}, 18 \mathrm{H}, 17 \mathrm{Ar}-\underline{\mathrm{H}}$ and1-N $\underline{\mathrm{H}}) .{ }^{13} \mathrm{C}$ NMR $(\delta \mathrm{ppm}, \mathrm{DMSO}): 39.2,46.0$, $62.9,66.7,109.2,111.4,112.5,114.6,116.3,120.8,121.9,124.0,126.3,129.7,132.2,134.4$, $136.9,142.0,144.3,152.5,164.6,166.8,173.5$.

Compound $\mathbf{A}_{8}: 2$ - (3' - trifluromethylphenylamino) - 4 - (tetrahydro - 1', 4' - oxazine) - 6 - [4' - \{1"- phenyl 5"- (3"' - phenoxyphenyl) $2^{\prime \prime}$ - pyrazolin $3^{\prime \prime}-$ yl $\}$ phenylamino] - s triazine : IR (KBr) cm $\mathrm{cm}^{-1}$ : $3376(-\mathrm{NH}), 2991$ (=CH str.), 2949 (C-H str., pyrazoline moiety), $1542\left(\mathrm{C}=\mathrm{N}\right.$, pyrazoline moiety), $1246(\mathrm{C}-\mathrm{O}-\mathrm{C}), 1049(\mathrm{C}-\mathrm{F}) .{ }^{1} \mathrm{H}$ NMR $\left(\delta \mathrm{ppm}, \mathrm{CDCl}_{3}\right): 2.9$ (dd, $\left.1 \mathrm{H},-\underline{\mathrm{C}}^{\mathrm{a}}-\mathrm{CH}-\right), 3.2$ (dd, $\left.1 \mathrm{H},-\underline{\mathrm{C}}^{\mathrm{b}}-\mathrm{CH}-\right), 4.8\left(\mathrm{dd}, 1 \mathrm{H},-\mathrm{C} \underline{\mathrm{H}}-\mathrm{CH}_{2}\right), 3.16\left(\mathrm{t}, 8 \mathrm{H},-\mathrm{CH}_{2}\right.$, 
oxazine ring), 6.9 to 8.6 (m, 23H, $22 \mathrm{Ar}-\underline{\mathrm{H} a n d 1-\mathrm{N}} \underline{\mathrm{H}}) .{ }^{13} \mathrm{C}$ NMR ( $\left.\delta \mathrm{ppm}, \mathrm{DMSO}\right): 40.0,48.6$, $61.0,64.9,110.5,112.4,114.3,117.0,119.6,121.8,123.1,125.7,127.0,128.3,130.0,133.5$, $141.4,143.8,149.7,156.2,157.1,163.4,165.0,174.9$.

Compound A9: 2 - (3' - trifluromethylphenylamino) - 4 - (tetrahydro - 1', 4' - oxazine) - 6 - [4' - \{1"- phenyl 5"- (2"' - nitrophenyl) $2 "$ - pyrazolin 3"- yl $\}$ phenylamino] - s - triazine : IR (KBr) cm ${ }^{-1}$ : $3302(-\mathrm{NH}), 3006$ (=CH str.), 3000 (C-H str., pyrazoline moiety), $1589(\mathrm{C}=\mathrm{N}$, pyrazoline moiety), 1486(C-NO $\left.\mathrm{NO}_{2}\right), 1231$ (C-O- C), $1061(\mathrm{C}-\mathrm{F}) .{ }^{1} \mathrm{H}$ NMR $\left(\delta \mathrm{ppm}, \mathrm{CDCl}_{3}\right)$ : $2.2\left(\mathrm{dd}, 1 \mathrm{H},-\underline{\mathrm{H}}^{\mathrm{a}}-\mathrm{CH}-\right), 2.5\left(\mathrm{dd}, 1 \mathrm{H},-\underline{\mathrm{H}}^{\mathrm{b}}-\mathrm{CH}-\right), 5.3\left(\mathrm{dd}, 1 \mathrm{H},-\mathrm{C} \underline{\mathrm{H}}-\mathrm{CH}_{2}\right), 3.46\left(\mathrm{t}, 8 \mathrm{H},-\mathrm{CH}_{2}\right.$,

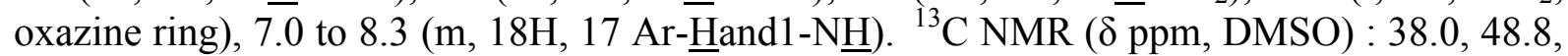
$65.7,66.2$, 111.2, 112.6, 115.0, 117.6, 118.4, 120.9, 122.2, 123.8, 126.1, 129.6, 131.9, 132.5, $140.7,142.0,145.1,150.9,163.0,166.4,173.8$.

Compound $\mathbf{A}_{10}: 2$ - (3' - trifluromethylphenylamino) - 4 - (tetrahydro - 1', 4' - oxazine) - 6 - [4' - \{1"- phenyl 5"- (phenyl) 2" - pyrazolin 3"- yl $\}$ phenylamino] - s - triazine : IR (KBr) $\mathrm{cm}^{-1}$ : $3398(-\mathrm{NH}), 3102(=\mathrm{CH}$ str.), 2965 (C-H str., pyrazoline moiety), 1560 (C=N, pyrazoline moiety), 1221 (C-O- C), $1098(\mathrm{C}-\mathrm{F}) .{ }^{1} \mathrm{H}$ NMR $\left(\delta \mathrm{ppm}, \mathrm{CDCl}_{3}\right): 2.4(\mathrm{dd}, 1 \mathrm{H}$, $\left.\underline{\mathrm{CH}}^{\mathrm{a}}-\mathrm{CH}-\right), 2.6$ (dd, $\left.1 \mathrm{H},-\underline{\mathrm{CH}}^{\mathrm{b}}-\mathrm{CH}-\right), 5.7$ (dd, $\left.1 \mathrm{H},-\mathrm{C} \underline{\mathrm{H}}-\mathrm{CH}_{2}\right), 3.78\left(\mathrm{t}, 8 \mathrm{H},-\mathrm{CH}_{2}\right.$, oxazine ring),

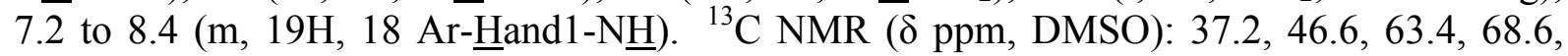
$108.5,110.2$, 112.4, 114.1, 116.6, 118.0, 120.5, 124.3, 127.0, 128.3, 130.7, 131.4, 141.2, $144.9,149.2,162.5,163.1,174.8$.

Compound $\mathbf{A}_{11}: 2$ - (3' - trifluromethyphenylamino) - 4 - (tetrahydro - 1', 4' - oxazine) - 6 - [4' - \{5"- (4"' - methoxyphenyl) $2 "$ - isoxazol - 3"- yl $\}$ phenylamino] - s - triazine : IR $(\mathrm{KBr}) \mathrm{cm}^{-1}$ : $3304(-\mathrm{NH}), 3089$ (=CH str.), $834 \quad(\mathrm{C}-\mathrm{H}$ bending), $1608 \quad(\mathrm{C}=\mathrm{N}$ str., isoxazolemoiety), $1253(\mathrm{C}-\mathrm{O}-\mathrm{C}), 1068(\mathrm{C}-\mathrm{F}) .{ }^{1} \mathrm{H}$ NMR $\left(\delta \mathrm{ppm}, \mathrm{CDCl}_{3}\right): 3.79(\mathrm{~s}, 3 \mathrm{H}$, p$\left.\mathrm{OC}_{3}\right), 3.56\left(\mathrm{t}, 8 \mathrm{H},-\mathrm{CH}_{2}\right.$, oxazine ring), $6.69(1 \mathrm{H}, \mathrm{s},-\mathrm{CH}=), 6.7$ to $8.1(\mathrm{~m}, 13 \mathrm{H}, 12 \mathrm{Ar}-\underline{\mathrm{H}}$ and1-N$).{ }^{13} \mathrm{C}$ NMR $(\delta$ ppm, DMSO) : 48.1, 56.7, 66.8, 98.4, 116.0, 116.9, 118.2, 121.3, 124.4, 127.0, 128.3, 129.8, 131.8, 138.9, 142.7, 160.6, 162.2, 165.7, 168.9, 169.3, 176.0.

Compound $\mathbf{A}_{12}: 2$ - (3' - trifluromethyphenylamino) - 4 - (tetrahydro - 1', 4' - oxazine) - 6 - [4' - \{5"- (4"' - chlorophenyl) $2 "$ - isoxazol - 3"- yl $\}$ phenylamino] - s - triazine : IR (KBr) $\mathrm{cm}^{-1}: 3373(-\mathrm{NH}), 3019$ (=CH str.), 815 (C-H bending), 1645 (C=N str., isoxazolemoiety), $1249(\mathrm{C}-\mathrm{O}-\mathrm{C}), 1066(\mathrm{C}-\mathrm{F}), 661(\mathrm{C}-\mathrm{Cl}) .{ }^{1} \mathrm{H}$ NMR $\left(\delta \mathrm{ppm}, \mathrm{CDCl}_{3}\right): 3.47\left(\mathrm{t}, 8 \mathrm{H},-\mathrm{CH}_{2}\right.$, oxazine ring), $6.56(1 \mathrm{H}, \mathrm{s},-\mathrm{CH}=), 6.9$ to $8.2(\mathrm{~m}, 13 \mathrm{H}, 12 \mathrm{Ar}-\underline{\mathrm{H}}$ and1-N$) .{ }^{13} \mathrm{C}$ NMR $(\delta \mathrm{ppm}$, DMSO) : 48.0, 66.8, 98.6, 112.1, 114.8, 120.6, 122.5, 123.7, 125.8, 127.9, 132.2, 133.8, 138.2, 142.9, 164.1, 166.3, 169.4, 173.2, 176.0.

Compound $\mathbf{A}_{13}: 2$ - (3' - trifluromethyphenylamino) - 4 - (tetrahydro - 1', 4' - oxazine) - 6 - [4' - \{5"- (3"' - phenoxyphenyl) $2 "$ - isoxazol - 3"- yl $\}$ phenylamino] - s - triazine : IR $(\mathrm{KBr}) \mathrm{cm}^{-1}: 3390(-\mathrm{NH}), 3080 \quad(=\mathrm{CH}$ str. $), 843 \quad(\mathrm{C}-\mathrm{H}$ bending), $1640 \quad(\mathrm{C}=\mathrm{N}$ str., isoxazolemoiety), $1250(\mathrm{C}-\mathrm{O}-\mathrm{C}), 1049(\mathrm{C}-\mathrm{F}),{ }^{1} \mathrm{H}$ NMR $\left(\delta \mathrm{ppm}, \mathrm{CDCl}_{3}\right): 3.72\left(\mathrm{t}, 8 \mathrm{H},-\mathrm{CH}_{2}\right.$,

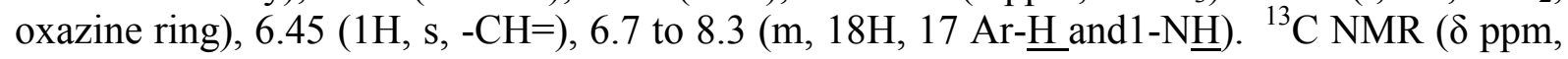
DMSO) : 47.9, 67.2, 99.4, 110.2, 112.5, 118.3, 121.4, 122.6, 124.2, 126.4, 127.0, 128.7, 132.1, 134.4, 137.2, 143.2, 156.7, 165.1, 168.8, 170.2, 171.5, 173.0.

Compound $\mathbf{A}_{14}: 2$ - (3' - trifluromethyphenylamino) - 4 - (tetrahydro - 1', 4' - oxazine) - 6 - [4' - \{5"- (2"' - nitrophenyl) $2 "$ - isoxazol - 3"- yl $\}$ phenylamino] - s - triazine : IR (KBr) $\mathrm{cm}^{-1}$ : $3311(-\mathrm{NH}), 3079$ (=CH str.), 824 (C-H bending), $1631(\mathrm{C}=\mathrm{N}$ str., isoxazolemoiety), 1481(C-NO $\left.{ }_{2}\right), 1228(\mathrm{C}-\mathrm{O}-\mathrm{C}), 1097(\mathrm{C}-\mathrm{F}) .{ }^{1} \mathrm{H}$ NMR $\left(\delta \mathrm{ppm}, \mathrm{CDCl}_{3}\right): 3.81\left(\mathrm{t}, 8 \mathrm{H},-\mathrm{CH}_{2}\right.$,

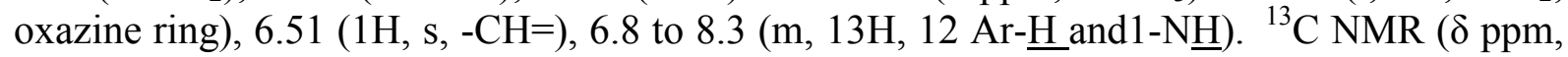
DMSO) : 48.7, 65.2, 96.4, 111.5, 115.7, 118.2, 121.0, 124.6, 126.5, 128.2, 131.4, 134.6, $137.0,141.1,146.8,165.8,167.5,170.6,172.1,174.5$. 
Compound $\mathbf{A}_{15}: 2$ - (3' - trifluromethyphenylamino) - 4 - (tetrahydro - 1', 4' - oxazine) - 6 - [4' - \{5"- (phenyl) 2" - isoxazol - 3"- yl $\}$ phenylamino] - s - triazine : IR $(\mathrm{KBr}) \mathrm{cm}^{-1}$ : 3356 (-NH), 3032 (=CH str.), 867(C-H bending), 1608 (C=N str., isoxazolemoiety), 1257 (CO- C), 1067 (C- F). ${ }^{1} \mathrm{H}$ NMR $\left(\delta \mathrm{ppm}, \mathrm{CDCl}_{3}\right): 3.24$ (t, $8 \mathrm{H},-\mathrm{CH}_{2}$, oxazine ring), $6.41(1 \mathrm{H}, \mathrm{s},-$ $\mathrm{CH}=), 6.8$ to $8.1(\mathrm{~m}, 14 \mathrm{H}, 13 \mathrm{Ar}-\underline{\mathrm{H}}$ and $1-\mathrm{N} \underline{\mathrm{H}}) .{ }^{13} \mathrm{C} \mathrm{NMR}(\delta \mathrm{ppm}, \mathrm{DMSO}): 46.1,66.3,98.2$, $112.5,114.1,120.3,123.1,125.8,127.2,129.0,131.7,133.2,138.4,142.8,164.6,166.4$, $169.0,172.7,174.2$.

\section{RESULT AND DISCUSSION}

\subsection{Antimicrobial evaluation}

All the newly synthesised compounds were screened for antibacterial and antifungal activity by Broth dilution method [30] against a panel of selected Gram - positive (S. aureus MTCC 96 and Streptococcus pyogenes MTCC 442) and Gram - negative bacteria (E. coli MTCC 443 and P. aeruginosa MTCC 441) and selected fungal strains (C. albicans MTCC 227, A. niger MTCC 282 and A calavatus MTCC 1323). DMSO was used as a solvent. Ampicillin and Chloramphenicol was used as a standard drug for antibacterial activity while Greseofulvin and Nystatin was used as a standard drug for antifungal activity. The results are showed in Table -2.

\subsection{A. Antibacterial Activity}

From the screening results (Table-2), it has been observed that, In Gram positive bacterial strains compounds $\mathrm{A}_{2}, \mathrm{~A}_{5}, \mathrm{~A}_{15}(\mathrm{MIC}=100 \mu \mathrm{g} / \mathrm{ml})$ and $\mathrm{A}_{8}, \mathrm{~A}_{9}, \mathrm{~A}_{10}(\mathrm{MIC}=125$ $\mu \mathrm{g} / \mathrm{ml}$ ) exhibited excellent activity, compounds $A_{1}, \quad A_{3}, A_{7}$ and $A_{11}(\mathrm{MIC}=200 \mu \mathrm{g} / \mathrm{ml})$ showed significant activity while compounds $A_{4}, A_{6}, A_{12}, A_{13}$ and $A_{14}(\mathrm{MIC}=250 \mu \mathrm{g} / \mathrm{ml})$ exhibited equipotent activity against S. aureus (MTCC 96) compared to Ampicillin (MIC = $250 \mu \mathrm{g} / \mathrm{ml})$. Compounds $A_{7}$ and $A_{10}(\mathrm{MIC}=100 \mu \mathrm{g} / \mathrm{ml})$ exhibited equipotent activity, whereas compounds $\mathrm{A}_{2}, \mathrm{~A}_{3}, \mathrm{~A}_{4}, \mathrm{~A}_{9}$ and $\mathrm{A}_{14}(\mathrm{MIC}=200 \mu \mathrm{g} / \mathrm{ml})$ were moderately active against $\mathrm{S}$. pyogenes (MTCC 442) compared to Ampicillin (MIC $=100 \mu \mathrm{g} / \mathrm{ml}$ ). In Gram negative bacterial strains compound $\mathrm{A}_{2}(\mathrm{MIC}=62.5 \mu \mathrm{g} / \mathrm{ml})$ exhibited excellent activity against $\mathrm{E}$. coli (MTCC 443) compared to Ampicillin ( $\mathrm{MIC}=100 \mu \mathrm{g} / \mathrm{ml}$ ) and modest to Chloramphenicol $(\mathrm{MIC}=50 \mu \mathrm{g} / \mathrm{ml})$ while compounds $\mathrm{A}_{3}, \mathrm{~A}_{7}, \mathrm{~A}_{8}(\mathrm{MIC}=100 \mu \mathrm{g} / \mathrm{ml})$ and $\mathrm{A}_{4}, \mathrm{~A}_{5}, \mathrm{~A}_{12}$ and $\mathrm{A}_{14}$ $(\mathrm{MIC}=125 \mu \mathrm{g} / \mathrm{ml}$ ) showed equipotent activity against $\mathrm{E}$. coli (MTCC 443) compared to Ampicillin $(\mathrm{MIC}=100 \mu \mathrm{g} / \mathrm{ml})$. Compound $\mathrm{A}_{7}(\mathrm{MIC}=62.5 \mu \mathrm{g} / \mathrm{ml})$ exhibited excellent activity against $\mathrm{P}$. aeruginosa (MTCC 441) compared to Ampicillin (MIC $=100 \mu \mathrm{g} / \mathrm{ml})$ and comparable to Chloramphenicol $\left(\mathrm{MIC}=50 \mu \mathrm{g} / \mathrm{ml}\right.$ ), whereas compounds $\mathrm{A}_{4}, \mathrm{~A}_{6}$ (MIC $=100$ $\mu \mathrm{g} / \mathrm{ml})$ and $\mathrm{A}_{2}, \mathrm{~A}_{12}(\mathrm{MIC}=125 \mu \mathrm{g} / \mathrm{ml})$ showed equipotent activity against $\mathrm{P}$. Aeruginosa (MTCC 441) compared to Ampicillin (MIC $=100 \mu \mathrm{g} / \mathrm{ml}$ ), while all other compounds were showed low to moderately active against all selected organisms.

\subsection{B. Antifungal Activity}

From the screening results (Table-2), it has been observed that, compound $\mathrm{A}_{2}$ (MIC $=$ $250 \mu \mathrm{g} / \mathrm{ml})$ and $\mathrm{A}_{7}(\mathrm{MIC}=100 \mu \mathrm{g} / \mathrm{ml})$ exhibited excellent activity against $\mathrm{C}$. albicans (MTCC 227) compared to Greseofulvin ( $\mathrm{MIC}=500 \mu \mathrm{g} / \mathrm{ml}$ ) and equipotent to Nystatin ( $\mathrm{MIC}=100$ 
$\mu \mathrm{g} / \mathrm{ml})$, while compounds $\mathrm{A}_{1}, \mathrm{~A}_{5}, \mathrm{~A}_{6}, \mathrm{~A}_{10}, \mathrm{~A}_{12}, \mathrm{~A}_{13}$ and $\mathrm{A}_{15}(\mathrm{MIC}=500 \mu \mathrm{g} / \mathrm{ml})$ showed comparable activity against $C$. Albicans (MTCC 227) compared to Greseofulvin (MIC $=500$ $\mu \mathrm{g} / \mathrm{ml})$. Compounds $\mathrm{A}_{1}(\mathrm{MIC}=200 \mu \mathrm{g} / \mathrm{ml})$ and $\mathrm{A}_{2}(\mathrm{MIC}=100 \mu \mathrm{g} / \mathrm{ml})$ exhibited equipotent activity against A. niger (MTCC 282) compared to Greseofulvin (MIC $=100 \mu \mathrm{g} / \mathrm{ml}$ ) and Nystatin (MIC $=100 \mu \mathrm{g} / \mathrm{ml}$ ). None of the compounds showed promising antifungal activity against A. clavatus (MTCC 1323).

Table 2. Antibacterial and antifungal activity data of compounds $A_{1}-A_{15}$.

\begin{tabular}{|c|c|c|c|c|c|c|c|}
\hline \multirow[b]{3}{*}{ Comp } & \multicolumn{4}{|c|}{ Minimal bactericidal concentration $\mu \mathrm{g} / \mathrm{ml}$} & \multirow{2}{*}{\multicolumn{3}{|c|}{ Minimal fungicidal concentration $\mu \mathrm{g} / \mathrm{ml}$}} \\
\hline & \multicolumn{2}{|c|}{ Gram positive } & \multicolumn{2}{|c|}{ Gram negative } & & & \\
\hline & $\begin{array}{c}\text { S. aureus } \\
\text { MTCC- } \\
96 \\
\end{array}$ & $\begin{array}{c}\text { S. } \\
\text { pyogenus } \\
\text { MTCC- } \\
442 \\
\end{array}$ & $\begin{array}{c}\text { E. coli } \\
\text { MTCC- } \\
443\end{array}$ & $\begin{array}{c}\text { P. aerug } \\
\text { MTCC- } \\
441\end{array}$ & $\begin{array}{c}\text { C. albicans } \\
\text { MTCC- } \\
227 \\
\end{array}$ & $\begin{array}{c}\text { A. niger } \\
\text { MTCC- } \\
282 \\
\end{array}$ & $\begin{array}{c}\text { A. clavatus } \\
\text { MTCC- } \\
1323 \\
\end{array}$ \\
\hline $\mathrm{A}_{1}$ & 200 & 250 & 200 & 250 & 500 & 200 & 500 \\
\hline $\mathrm{A}_{2}$ & 100 & 200 & 62.5 & 125 & 250 & 100 & 500 \\
\hline $\mathrm{A}_{3}$ & 200 & 200 & 100 & 200 & 1000 & 500 & 500 \\
\hline $\mathrm{A}_{4}$ & 250 & 200 & 125 & 100 & 1000 & 500 & $>1000$ \\
\hline $\mathrm{A}_{5}$ & 100 & 250 & 125 & 200 & 500 & 1000 & $>1000$ \\
\hline $\mathrm{A}_{6}$ & 250 & 250 & 200 & 100 & 500 & 1000 & 1000 \\
\hline $\mathrm{A}_{7}$ & 200 & 100 & 100 & 62.5 & 100 & 1000 & 500 \\
\hline $\mathrm{A}_{8}$ & 125 & 250 & 100 & 250 & 1000 & 1000 & 1000 \\
\hline $\mathrm{A}_{9}$ & 125 & 200 & 250 & 500 & 1000 & 1000 & 1000 \\
\hline $\mathrm{A}_{10}$ & 125 & 100 & 200 & 250 & 500 & $>1000$ & $>1000$ \\
\hline $\mathrm{A}_{11}$ & 200 & 500 & 200 & 200 & 1000 & 500 & 1000 \\
\hline $\mathrm{A}_{12}$ & 250 & 250 & 125 & 125 & 500 & 250 & 250 \\
\hline $\mathrm{A}_{13}$ & 250 & 250 & 500 & 500 & 500 & 500 & 500 \\
\hline $\mathrm{A}_{14}$ & 250 & 200 & 125 & 250 & 1000 & $>1000$ & $>1000$ \\
\hline $\mathrm{A}_{15}$ & 100 & 250 & 250 & 250 & 500 & 1000 & 1000 \\
\hline $\mathrm{A}$ & 250 & 100 & 100 & 100 & - & - & - \\
\hline $\mathrm{B}$ & 50 & 50 & 50 & 50 & - & - & - \\
\hline $\mathrm{C}$ & - & - & - & - & 500 & 100 & 100 \\
\hline $\mathrm{D}$ & - & - & - & - & 100 & 100 & 100 \\
\hline
\end{tabular}

Where $\mathrm{A}=$ Ampicillin, $\mathrm{B}=$ Chloramphenicol (Standard Drugs for antibacterial activity)

$\mathrm{C}=$ Greseofulvin, $\mathrm{D}=$ Nystatin (Standard Drugs for antifungal activity)

\section{CONCLUSION}

In outline, we have synthesised some bioactive chalcones and convert them into pyrazoline and isoxazole moiety by using conventional method. The method adopted for the synthesis of pharmacologically important molecules in this investigation is simple, efficient, and inexpensive. The IR, ${ }^{1} \mathrm{H}$ NMR, ${ }^{13} \mathrm{C}$ NMR spectral analysis and elemental analysis of all 
the newly synthesised compounds confirmed that purity of the entire synthesised compound is good.

All the synthesised compounds were screened for antimicrobial activity. Majority of the synthesised compounds were found to potentially active against both selected Gram positive, Gram negative organisms and selected fungal organisms. From the results of antibacterial and antifungal activity, it can be concluded that compounds $\mathbf{A}_{\mathbf{2}}, \quad \mathbf{A}_{\mathbf{7}}$ and $\mathbf{A}_{\mathbf{1 2}}$ were found more active then the remaining compounds due to the present of chlorine atom. So overall it was revealed that the no substitution on phenyl ring showed no inhibition of the tested bacteria while the compounds that showed some inhibition was due to the presence of substitution of methoxy, chloro, phenoxy and nitro group on some position of the phenyl ring. These finding conclued that the titled compounds have the properties to kill the microbes in some extent when compared with standared drug. These result suggest that chalcone and their derivatives have an opportunity to behave as broad spectrum antimicrobial agents and have exellent scope for further development as commercial antimicrobial agents .

\section{Acknowledgement}

Authors are grateful to B. K. M. Science College, Valsad for providing research facilities, Atul Ltd. (Atul) for the IR analysis, RSIC Punjab University for the ${ }^{1} \mathrm{H}-\mathrm{NMR},{ }^{13} \mathrm{C}$ NMR spectral analysis as well as elemental analysis and Microcare Laboratory, Surat, for antimicrobial activity screening.

\section{References}

[1] J. Quiroga, Y. Diaz, B. Insuasty, R. Abonia, M. Nogueras, J. Cobo, Tetrahedron Letters 51 (21) (2010) 2928 - 2930.

[2] N. Sunduru, Nishi, S. Palne, P. M. S. Chavhan, S. Gupta, European Journal of Medicinal Chemistry 44 (6) (2009) 2473 - 2481.

[3] T. Shah, V. Desai, Journal of Serbian Chemical Society 72 (5) (2007) 443 - 449.

[4] N. Kumar, S. Tiwari, A. K. Yadav, Indian Journal of Chemistry 46B (4) (2007) 702 706.

[5] W. J. Zhou, S. J. Ji, Z. L. Shen, Journal of Organometallic Chemistry 691 (7) (2006) $1356-1360$.

[6] P. S. Patil, S. M. Dharmaprakash, K. Ramakrishna, H. K. Fun, R. S. S. Kumar, D. N. Rao, Journal of Crystal Growth 303 (2) (2007) 520 - 524.

[7] M. T. Konieczny, W. Konieczny, M. Sabisz, A. Skladanowski, R. Wakiec, K. E. Augustynowicz, Z. Zwolska, Europian journal of Medicinal Chemistry 42 (5) (2007) $729-733$.

[8] D. Kumar, N. M. Kumar, K. Akamatsu, E. Kusaka, H. Harada, T. Ito, Bioorganic Medicinal Chemistry Letters 20 (13) (2010) 3916 - 3919.

[9] S. Ducki, R. Forrest, J. A. Hadfield, A. Kendall, N. J. Lawrence, A. T. McGown, D. Rennison, Bioorganic Medicinal Chemistry Letters 8 (9) (1998) 1051 - 1056.

[10] Z. Nowakowska, European Journal of Medicinal Chemistry 42 (2) (2007) 125 - 137. 
[11] O. Sabzevari, S. Mahmoudian, B. Minaei, H. Paydar, Toxicology Letters 196 (2010) S213.

[12] F. F. Barsoum, H. M. Hosni, A. S. Girgis Bioorganic Medicinal Chemistry 14 (11) (2006) 3929 - 3937.

[13] R. Foresti, M. Hoque, D. Monti, C. J. Green, R. Motterlini, Journal of Pharmacology and Experimental Therapeutics 312 (2) (2004) 686 - 693.

[14] N. S. Mewada, D. R. Shah, K. H. Chikhalia, International Letters of Chemistry, Physics and Astronomy 17 (3) (2014) 281- 294.

[15] X. H. Zhang, S. K. Wu, Z. Q. Gao, C. S. Lee, S. T Lee, H. L. Kwong, Thin Solid Films $371(1$ - 2) (2000) 40 - 46.

[16] G. Thirunarayanan, International Letters of Chemistry, Physics and Astronomy 18 (2014) 47-56.

[17] M. Amir, H. Kumar, S. A. Khan, Bioorganic and Medicinal Chemistry Letters 18 (3) (2008) 918 - 922.

[18] M. I. Hussain, S. Shukla, Indian Journal of Chemistry 25B (1986) 983 - 986.

[19] A. A. Bilgin, E. Palaska, R. Sunal, and B. Gunnesel, Pharmazie 49 (1) (1994) 67 - 69.

[20] A. Solankee, S. Solankee, G. Patel, K. Patel, R. Patel, Der Pharma Chemica 3 (1) (2011) $300-305$.

[21] M. Shekarchi, B. P. Hamedani, L. Navidpour, N. Adib, A. Shafiee, Journal of Iranian Chemical Society 5 (1) (2008) 150 - 158.

[22] J. Deng, T. Sanchez, N. Neamati, J. M. Briggs, Journal of Medicinal Chemistry 49 (5) (2006) 1684 - 1692.

[23] B. J. Wakefield, D. J. Wright, Advance Heterocyclic Chemistry 25 (1979) 147 - 186.

[24] C. Kashima, Heterocycles 12 (10) (1979) 1343 - 1368.

[25] M. M. M. Santos, F. Natalia, I. Jim, C. J. Simon, H. B. Michael, M. L. Martins, M. Rui, Bioorganic Medicinal Chemistry Letters 20 (1) (2010) 193 -195.

[26] A. Solankee, K. Patel, R. Patel, Elixir Organic Chemistry 44 (2012) 7316 - 7319.

[27] V. Subash, B. Michael, U. Reaz, W. Baojie, F. G. Scott, P. A. Pavel, Journal of Medicinal Chemistry 51 (10) (2008) 1999 -2002.

[28] J. A. Egan, R. P. Nugent, C. N. Filer, Journal of Radioanalytical and Nuclear Chemistry 279 (3) (2009) 935 - 936.

[29] A. Solankee, K. Kapadia, Anaciric, M. Sokovic, I. Doytchinova, A. Geronikaki, Europian Journal of medicinal chemistry 45 (2) (2010) 510 - 518.

[30] A. Rattan, 5th ed. B. Y. Churchill Livingstone (2005) 85 - 90. 